Signal \& Image Processing : An International Journal (SIPIJ) Vol.5, No.1, February 2014

\title{
A STUDY OF A MODIFIED HISTOGRAM BASED FAST ENHANCEMENT ALGORITHM (MHBFE)
}

\author{
Amany A. Kandeel ${ }^{1}$, Alaa M. Abbas ${ }^{2,4}$, Mohiy M. Hadhoud ${ }^{3}$, Zeiad El-Saghir ${ }^{1}$ \\ ${ }^{1}$ Dept. of Computer Science and Engineering, Faculty of Electronic Engineering, \\ Univ. of Menoufia, Egypt. \\ ${ }^{2}$ Dept. Of Electronics and Electrical Communications, \\ Faculty of Electronic Engineering, Univ. of Menoufia, Egypt. \\ ${ }^{3}$ Dept. of Information Technology, Faculty of Computers and Information, \\ Univ. of Menoufia, Egypt. \\ ${ }^{4}$ Dept. of Electrical Engineering, Faculty of Engineering, Taif University, Saudi Arabia
}

\begin{abstract}
Image enhancement is one of the most important issues in low-level image processing. The goal of image enhancement is to improve the quality of an image such that enhanced image is better than the original image. Conventional Histogram equalization $(\mathrm{HE})$ is one of the most algorithms used in the contrast enhancement of medical images, this due to its simplicity and effectiveness. However, it causes the unnatural look and visual artefacts, where it tends to change the brightness of an images. The Histogram Based Fast Enhancement Algorithm (HBFE) tries to enhance the CT head images, where it improves the water-washed effect caused by conventional histogram equalization algorithms with less complexity. It depends on using full gray levels to enhance the soft tissues ignoring other image details. We present a modification of this algorithm to be valid for most CT image types with keeping the degree of simplicity. Experimental results show that The Modified Histogram Based Fast Enhancement Algorithm (MHBFE) enhances the results in term of PSNR, AMBE and entropy. We use also the Statistical analysis to ensure the improvement of the proposed modification that can be generalized. ANalysis Of VAriance (ANOVA) is used as first to test whether or not all the results have the same average. Then we find the significant improvement of the modification.
\end{abstract}

\section{KEYWORDS}

Histogram equalization (HE), Contrast enhancement, Histogram Based Fast Enhancement Algorithm (HBFE), CT image, Modified Histogram Based Fast Enhancement Algorithm (MHBFE), ANalysis Of VAriance (ANOVA)

\section{INTRODUCTION}

Medical images plays an important role in diagnosing diseases, where doctors use it as the first step for the diseases recognition. They detect any pathological changes from organs scans [1]. Not only, it is used in diagnosis but also it can help in reducing mortality rate. This occur by

DOI : $10.5121 /$ sipij.2014.5105 
Signal \& Image Processing : An International Journal (SIPIJ) Vol.5, No.1, February 2014

improving earlier detection and treatment stages, in which the time factor is very important to discover the disease in the patient as possible as fast, especially in various cancer tumours such as the lung cancer and the breast cancer [2,3]. There are many medical imaging techniques, Computed tomography (CT) is considered as the most popular of them after developed in 1970's [4].The major factors affected the medical image quality are Noise and resolution. Many image enhancement algorithms used to reduce these factors influence [5].

Histogram Equalization (HE) is considered as the most popular algorithm for contrast enhancement. Its basic idea lies in mapping the gray levels based on the probability distribution of the input gray levels. It flattens and stretches the dynamic range of the image's histogram, resulting in an overall contrast improvement. HE has been applied in various fields such as medical image processing and radar image processing [6, 7]. The two categories of histogram equalization are: Global histogram equalization, which is simple and fast, but its contrastenhancement power is relatively low. Local histogram equalization, on the other hand, can effectively enhance contrast, but it requires more computations.

Global Histogram equalization is powerful in highlighting the borders and edges between different objects, but may reduce the local details within these objects to overcome HE's problems [8]. Ketcham and et al invented Local Histogram Equalization (LHE); LHE uses the histogram of a window of a predetermined size to determine the transformation of each pixel in the image. LHE succeeded in enhancing local details, but it depends on fixed size for windows where it may distort the boundaries between regions. It also demands high computational cost and sometimes causes over-enhancement in some portion of the image $[9,10]$.

There are many algorithms try to preserve the brightness of the output image like Brightness preserving Bi-Histogram Equalization (BBHE) which separates the input image histogram into two parts based on the mean of the input image and then each part is equalized independently. There are many methods similar to BBHE like Dualistic Sub-Image Histogram Equalization (DSIHE), where it divides the histogram based on the median value. Modified Dualistic Sub Image Histogram Equalization (MDSIHE), A. Zadbuke made a modification on DSIHE and obtained good results [11]. Minimum Mean Brightness Error Bi-Histogram Equalization (MMBEBHE) provides maximal brightness preservation, but its results are found not good for the image with a lot details. To overcome these drawbacks, P. Jagatheeswari and et al proposed a modification to this method. They enhanced images by passing the enhanced ones through a median filter. The median filter is an effective method for the removal of impulse based noise on the images [12]. Recursive Mean-Separate Histogram Equalization (RMSHE) is also considered as an extension to BBHE. All these methods achieve good contrast but they have some problems in gray level variation [10].

There are many algorithms start to combine the features of the local and global techniques. H. Cheng and et al improve the global histogram equalization by using multi-peak histogram equalization combined with local information, their algorithm success to enhance various kinds of images when the proper features (local information) can be extracted [13]. J.Kim and et al keep the high contrast of local histogram equalization with the simplicity of global histogram equalization. Their algorithm computation overhead is reduced by a factor of about 100 compared to that of local histogram equalization [14]. 
Signal \& Image Processing : An International Journal (SIPIJ) Vol.5, No.1, February 2014

The rest of the paper is organized as follows in section 2, the idea of A Histogram-Based Fast Enhancement Algorithm will be introduced. Then, the problems were found in this algorithm and the suggested modification is presented in section 3. Experimental results using clinical data of CT images is discussed in section 4 to demonstrate the usefulness of the proposed method. The Statistical analysis of the proposed algorithm is illustrated in section 5 with showing the different methods are used. At the last, concluding remarks is presented in section 6 .

\section{A HiStOGRAM BASED FAST ENHANCEMENT ALgORITHM (HBFE)}

J. Yin and et al proposed an algorithm to enhance the interested areas in CT head images; they tried to improve the water-washed effect caused by the conventional histogram equalization algorithms as shown in Figure 1. We will give it abbreviation of HBFE in this paper. The algorithm succeeded in removing water-washed effect. There are some important features for this algorithm like the speed and the simplicity. Its idea depends on that, most CT head images occupy the gray level 0 , so they try to deal with the soft tissues by enhancing the region by using full range of all possible gray levels to enhance it in the CT head images. They analysed these images and found that more than half of the whole range of gray levels occupies 0 level, and all CT head images have three major peaks in their histograms. The left peak is formed by background pixels, the middle peak is usually formed by soft tissues in the CT head images, and the right peak is formed mostly by bone. For enhancement details, only the middle peak which formed by soft tissue is needed [15].

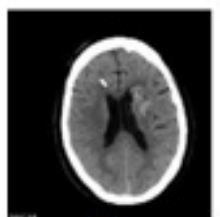

(a)

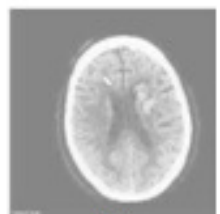

(b)

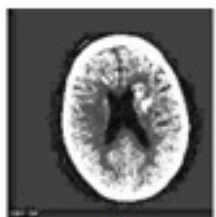

(c)

Figure 1. (a) An original CT head image (b) enhanced by conventional histogram equalization algorithm (c) Histogram-Based Fast Enhancement Algorithm.

\section{A Modified Histogram BASED FAST ENHANCEMENT ALGORITHM (MHBFE)}

The idea of HBFE Algorithm is based on the characteristics of CT head images. This makes the algorithm is suitable for special type of images, so we try to make a modification to this algorithm to be more appropriate for a wide range of CT images with enhanced results. The calculations of the algorithm depends on a constant value $\mathrm{k}(0<\mathrm{k}<0.4)$ to evaluate how many gray levels should be ignored. This means that $\mathrm{k}$ remains constant for all images regardless of image characteristics, so we calculate the value of $\mathrm{k}$ to be variable according to the features image's gray levels.

First, we evaluate $\mathrm{k}$ as a ratio of the mean value of histogram values, which is considered as an important feature of the histogram then we recorded these results, and compared it with the HBFE; we found that there is a valuable enhancement in results. The steps of our proposed solution remained as in HBFE Algorithm, but the change will be occurred in determining $\mathrm{k}$ value as below: 
Signal \& Image Processing : An International Journal (SIPIJ) Vol.5, No.1, February 2014

$$
k=k_{c} * H_{\text {mean }}
$$

Where $\mathrm{k}_{\mathrm{c}}$ is a constant value we determined form experimental results to achieve the best values, $\mathrm{H}_{\text {mean }}$ is the mean value of the histogram, which is the sum of the histogram values divided by the number of histogram bins.it evaluated from the following equation:

$$
H_{\text {mean }}=\frac{\sum_{i=1}^{n} H(i)}{n}
$$

Where H (i) is the repetitions' count of $i$ bins of gray levels, $n$ is the number of gray levels of the image. As known that the mean value is one of the most features of the image parameters, where it represent the distribution of image's gray levels. So we select this parameter to determine the number of gray levels will be ignored to complete the reminder algorithm steps. After applying the calculation of mean, we find that there is an enhancement on the three image' parameters of PSNR (Peak Signal-to-Noise Ratio), AMBE (Absolute Mean Brightness Error) and Entropy. The results will be shown in the next section.

After that we study another parameter that is the median, we performed the modification by using $\mathrm{k}$ as a ratio of median value of the histogram and found that the results become better that because this value also depends on the characteristic of image.

$$
k=k_{c} * H_{\text {median }}
$$

Where $\mathrm{H}_{\text {median }}$ is the median value of the histogram, it is defined as the value which divides the values into two equal halves. It also achieve more enhancement in the PSNR, Entropy and AMBE. At the last, we use the mode value $\mathrm{H}_{\text {mode }}$ which is the most frequently occurring value in the histogram.

$$
k=k_{c} * H_{\text {mode }}
$$

The median value is considered as the best proposed modification for the algorithm, the compared results will be discussed later. We applied the modified algorithm to large varieties of CT images including head and lung images. To evaluate the effectiveness of the modification we use three widely-used metrics; PSNR, AMBE, and the entropy. We will show briefly how to evaluate these metrics in the next section.

\subsection{Peak Signal to Noise Ratio (PSNR)}

PSNR is the evaluation standard of the reconstructed image quality, and is an important measurement feature. PSNR is measured in decibels $(\mathrm{dB})$. If we suppose a reference image $f$ and a test image $t$, both of size $M \times N$, the PSNR between $f$ and $t$ is defined by:

$$
\operatorname{PSNR}(f, t)=\log _{10}(L-1)^{2} / M S E(f, t)
$$

Where $\mathrm{L}$ is gray levels and MSE (Mean square error) is calculated as: 
Signal \& Image Processing : An International Journal (SIPIJ) Vol.5, No.1, February 2014

$$
\operatorname{MSE}(f, t)=\frac{1}{M N} \sum_{i=1}^{M} \sum_{j=1}^{N}\left(f_{i j}-t_{i j}\right)^{2}
$$

Note that the greater the PSNR, the better the output image quality.

\subsection{Absolute Mean Brightness Error (AMBE)}

It is the difference between original and enhanced image and is given as:

$$
\operatorname{AMBE}(X, Y)=|X M-Y M|
$$

Where $X M$ is the mean of the input image $X=\{X(i, j)\}$ and $Y M$ is the mean of the output image $\mathrm{Y}=\{\mathrm{Y}(\mathrm{i}, \mathrm{j})\}$. We try to preserve the brightness of the image to keep the image details, so if we reduce the difference this preserve the brightness of the image.

\subsection{Entropy}

Entropy is a statistical measure of randomness that can be used to characterize the texture of the input image. It is a useful tool to measure the Richness of the details in the output image [16].

$$
\operatorname{Ent}[P]=\sum_{i=1}^{n}\left(P_{i} \log _{2}\left(P_{i}\right)\right)
$$

Where $\mathrm{P}_{\mathrm{i}}$ is the probability of the occurrence of symbol $\mathrm{i}$.

\subsection{Inspection of Visual Quality}

In addition to the quantitative evaluation of contrast enhancement using the PSNR and entropy values, it is also important to qualitatively assess the contrast enhancement. The major goal of the qualitative assessment is to judge if the output image is visually acceptable to human eyes and has a natural appearance [11].

\section{EXPERIMENTAL RESULTS}

To show the enhancement that occurred using the MHBFE, we apply it on a different types of CT images. However, HBFE applied only on CT head images. We use CT lung and head images. This is to be validate for all types of CT images. 
Signal \& Image Processing : An International Journal (SIPIJ) Vol.5, No.1, February 2014

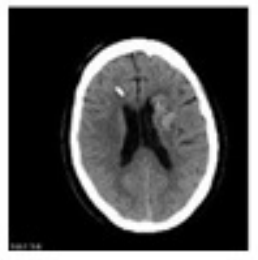

(a)

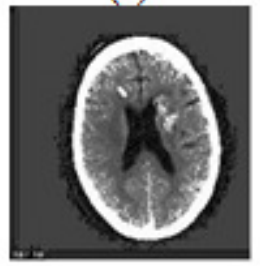

(d)

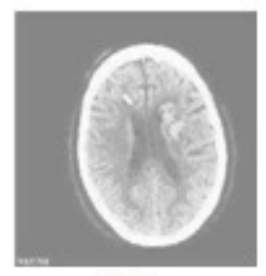

(b)

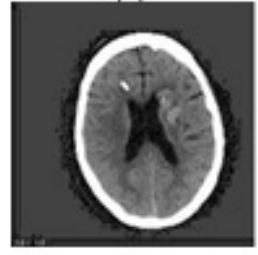

(e)

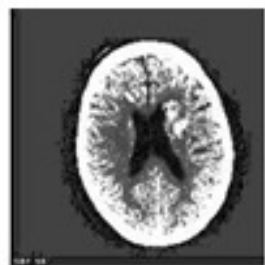

(c)

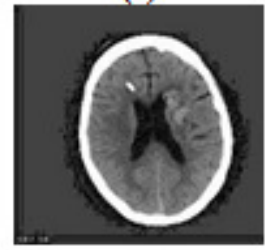

(f)

Figure 2. (a) Original CT head image (b) enhanced by conventional histogram equalization algorithm (c) enhanced by HBFE (d) MHBFE using mean value (e) MHBFE using median value (f) MHBFE using mode value

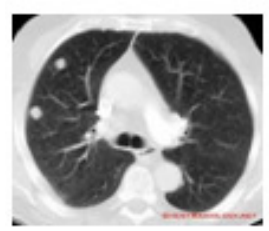

(a)

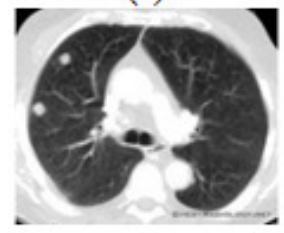

(d)

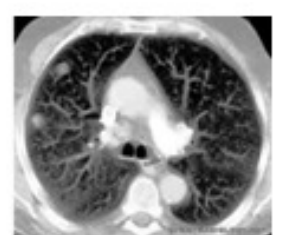

(b)

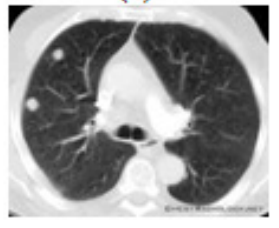

(e)

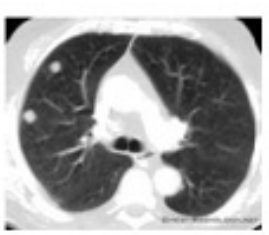

(c)

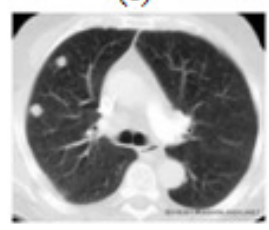

(f)

Figure 3. (a) Original CT lung image (b) enhanced by conventional histogram equalization algorithm (c) enhanced by HBFE (d) MHBFE using mean value (e) MHBFE using median value (f) MHBFE using mode value

We will mention only 8 images in the following tables regards to be clearly explained; 4 CT head and $4 \mathrm{CT}$ lung. In the next section, large number of images will be used.

As we mention before, the increase in the value of PSNR is considered as an enhancement in the algorithm. This is due to the decrement of noise ratio in the enhanced image. Table 1 contain the results PSNR measured using the HE, HBFE and MHBFE with three of modifications (mean, median and mode). As we see in Table 1 we find that there is an improvement in PSNR values using the proposed modified algorithm with its three methods. 
Signal \& Image Processing : An International Journal (SIPIJ) Vol.5, No.1, February 2014

Table 1. PSNR measurement.

\begin{tabular}{|c|c|c|c|c|c|}
\hline \multirow{2}{*}{ Image } & \multirow{2}{*}{ HE } & \multirow{2}{*}{ HBFE } & \multicolumn{3}{|c|}{ MHBFE } \\
\cline { 4 - 6 } & & & Using Mean & Using Median & Using Mode \\
\hline CThead1 & 6.658 & 12.168 & 13.953 & 14.632 & 14.632 \\
\hline CThead2 & 6.718 & 12.310 & 14.815 & 14.817 & 14.817 \\
\hline CThead3 & 4.278 & 9.020 & 9.808 & 11.222 & 11.222 \\
\hline CThead4 & 10.019 & 17.035 & 18.956 & 27.541 & 34.349 \\
\hline CTlung1 & 17.969 & 26.984 & 28.610 & 32.356 & 34.249 \\
\hline CTlung2 & 19.318 & 30.142 & 32.392 & 41.849 & 43.584 \\
\hline CTlung3 & 8.839 & 13.835 & 13.464 & 14.504 & 14.505 \\
\hline CTlung4 & 18.316 & 25.973 & 27.398 & 30.386 & 30.601 \\
\hline
\end{tabular}

Table 2. AMBE measurement.

\begin{tabular}{|c|c|c|c|c|c|}
\hline \multirow{2}{*}{ Image } & \multirow{2}{*}{ HE } & \multirow{2}{*}{ HBFE } & \multicolumn{3}{|c|}{ MHBFE } \\
\cline { 4 - 6 } & & & Using Mean & Using Median & Using Mode \\
\hline CThead1 & 111.870 & 48.143 & 38.046 & 34.558 & 34.558 \\
\hline CThead2 & 97.365 & 41.379 & 133.749 & 30.063 & 13.385 \\
\hline CThead3 & 150.441 & 78.696 & 70.721 & 57.666 & 57.666 \\
\hline CThead4 & 72.255 & 13.667 & 10.430 & 4.334 & 3.074 \\
\hline CTlung1 & 13.457 & 4.982 & 3.427 & 1.969 & 1.646 \\
\hline CTlung2 & 15.107 & 4.1489 & 3.235 & 1.552 & 1.369 \\
\hline CTlung3 & 76.996 & 41.857 & 43.534 & 35.296 & 35.262 \\
\hline CTlung4 & 26.138 & 6.898 & 5.876 & 4.1484 & 3.992 \\
\hline
\end{tabular}

Our Proposed algorithm is considered one of brightness persevered algorithm so we try to reduce the difference between the brightness of input and the result image. From Table 2, we can conclude that there is a clear enhancement in AMBE values using the proposed algorithm. As shown in the table, the enhancement value depends on the images.

As we will see in Table 3, there is a small increase in the Entropy values. We notice that the results of MHBFE using the median and the mode in some images are very similar, where we have found there is a great convergence between their values.

Table 3. Entropy measurement

\begin{tabular}{|c|c|c|c|c|c|c|}
\hline Image & \multirow{2}{*}{$\begin{array}{c}\text { Original } \\
\text { Image }\end{array}$} & HE & HBFE & \multicolumn{3}{|c|}{ MHBFE } \\
\cline { 5 - 7 } & & & Using Mean & Using Median & Using Mode \\
\hline CThead1 & 4.310 & 3.323 & 4.608 & 4.912 & 5.133 & 5.133 \\
\hline CThead2 & 5.832 & 4.601 & 5.144 & 5.195 & 5.391 & 5.838 \\
\hline CThead3 & 3.200 & 2.229 & 2.812 & 2.997 & 3.377 & 3.377 \\
\hline CThead4 & 6.495 & 5.478 & 6.323 & 6.399 & 6.587 & 6.633 \\
\hline CTlung1 & 6.997 & 5.897 & 6.924 & 7.085 & 7.102 & 7.045 \\
\hline CTlung2 & 7.234 & 5.952 & 6.842 & 6.927 & 7.162 & 7.202 \\
\hline CTlung3 & 4.274 & 3.320 & 3.517 & 3.409 & 4.483 & 4.500 \\
\hline CTlung4 & 7.277 & 5.389 & 6.731 & 6.782 & 6.894 & 6.906 \\
\hline
\end{tabular}


Signal \& Image Processing : An International Journal (SIPIJ) Vol.5, No.1, February 2014

As for the Inspection of Visual Quality, as we see in Figure 2 and Figure 3 there are some details appeared in the proposed algorithm which help in diagnostic diseases more accurate.

We can exclude some points from the previous results that the modified algorithm achieves greater values of PSNR, AMBE and entropy compared with HBFE. The first metric of PSNR; the proposed algorithm have increased the values of PSNR; this means that less noise in the resulted image. The second metric is AMBE, it has been minimized and this means that it has preserved the brightness of the image. The third metric of entropy where it has increased; this means that more information can be extracted from the output image. To estimate the constant value used in the calculations, we perform the algorithms with rang of constant values then summarize the results in Figure 4, Figure 5, and Figure 6, where Figure 4 shows the increment in PSNR values due to using the modification with mean, median and mode. Figure5 shows the enhancement in entropy values and Figure 6 show the decrement of AMBE. There is a valuable improvement in the three parameters for the modification especially the mode where give the best results. We found that there is a range of the constant values that gives the best results for the three parameters and outside this range there are less improved results. This gives us the ability to control this ratio to obtain the best results.

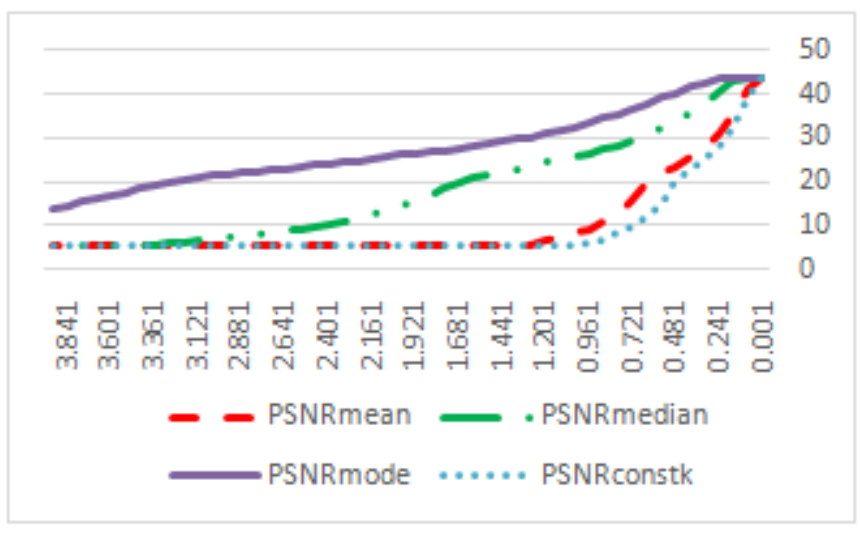

Figure 4. The effect of modification on PSNR values

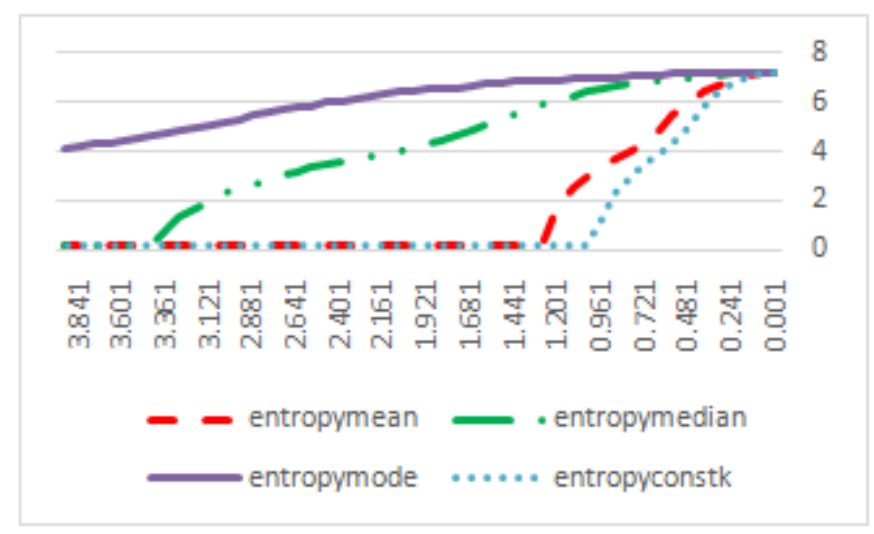

Figure 5. The effect of modification on entropy values 
Signal \& Image Processing : An International Journal (SIPIJ) Vol.5, No.1, February 2014

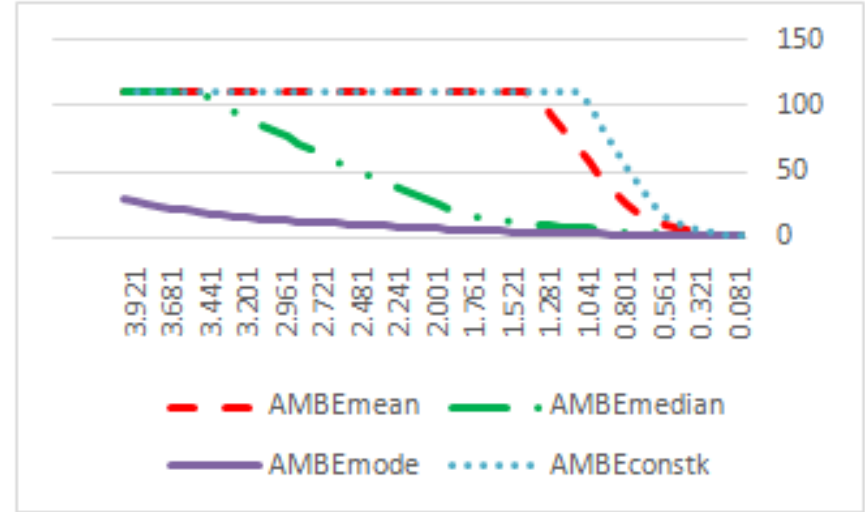

Figure 6. The effect of modification on AMBE values

\section{STATISTICAL ANALYSIS}

In the previous section, the experimental results show that the modification achieves enhancement in the three parameters of PSNR, Entropy and AMBE. But the sample size used in the previous section is small and this is for more clearly in illustration the results, but to make the results to be more generalized the statistical analysis should be performed on larger data sample. So in this section; we will use statistical analysis to find the significance enhancement of the proposed modification. We use the hypothesis testing approach which has a detailed protocols for decision-making concerning a population by examining a sample from that population [17]. There are two assumptions that should be satisfied before using the hypothesis testing; 1) the sample of images should be randomly selected, 2) the sample data should come from approximately normal distribution.

To satisfy these assumptions we randomly select different CT lung and head image as a sample data. This achieves the first assumption. To satisfy the second assumption of the normality, the population distribution of the sample is drawn. It should be normal or approximately normal. We draw the distribution graphs for the three parameters (PSNR, entropy and AMBE) of the HBFE and MHBFE with the three modification. The normal quantile plot will be created to check the normality assumption as shown in figure 10. The assumption is met if the points fall close to the red line [17].

After draw the normality graph, we calculate the normality percentage. The Goodness-of-fit parameter is considered as a measure for the normality, which is calculated using Shapiro-wilk W test, which calculates a W statistic as:

$$
\mathrm{W}=\frac{\left(\sum_{\mathrm{i}=1}^{\mathrm{n}} \mathrm{a}_{\mathrm{i}} \mathrm{x}_{(\mathrm{i})}\right)^{2}}{\sum_{\mathrm{i}=1}^{\mathrm{n}}\left(\mathrm{x}_{\mathrm{i}}-\overline{\mathrm{x}}\right)^{2}}
$$

Where the $x_{(i)}$ are the ordered sample values $\left(x_{(1)}\right.$ is the smallest) and the $a_{i}$ are constants generated from the means, variances and covariance of the order statistics of a sample of size $n$ from a normal distribution. It tests whether a random sample $x_{1}, x_{2} \ldots x_{n}$ comes from (specifically) a normal distribution. Small values of $W$ are evidence of departure from normality [18]. The calculated Goodness-of-fit values are summered in Table 4. 
Signal \& Image Processing : An International Journal (SIPIJ) Vol.5, No.1, February 2014
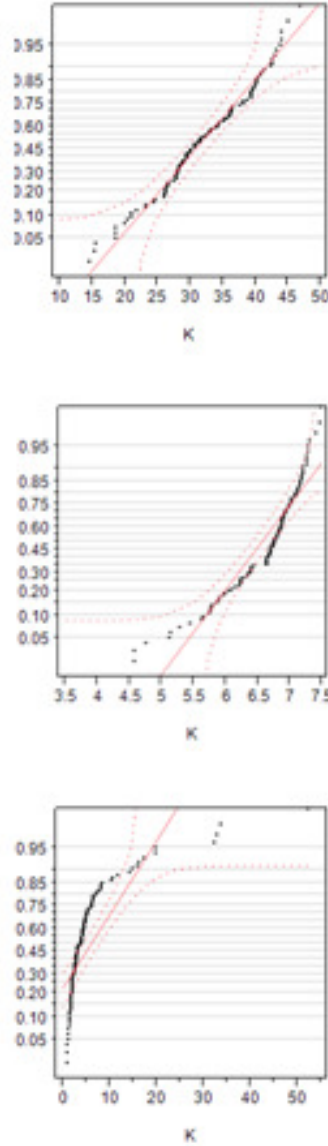

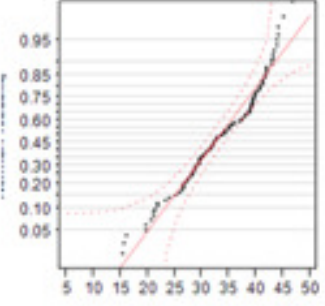

Nean
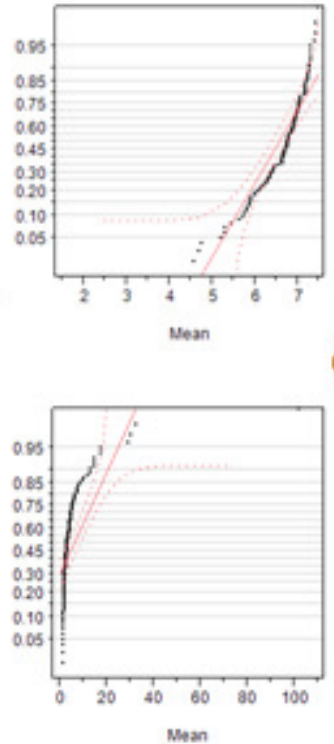

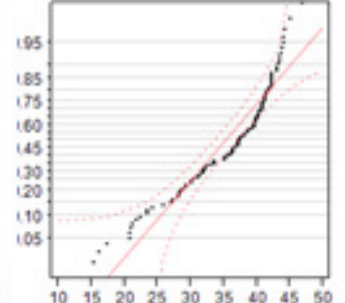

Mesan

(a)

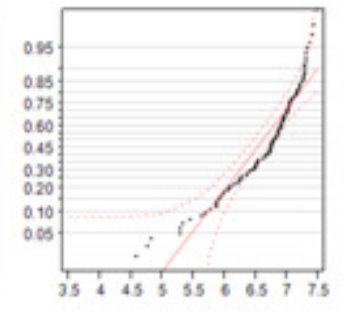

Mosan

(b)

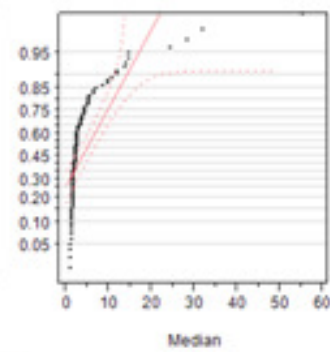

(c)

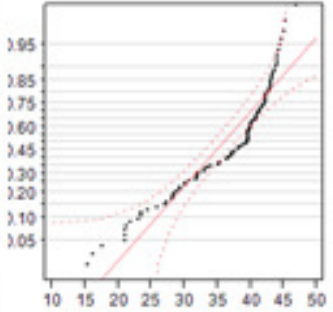

Mose
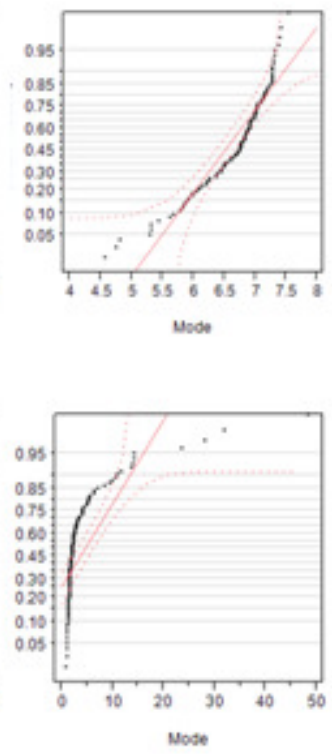

Figure 10: The normal quantile plot of the HBFE with constant k, MHBFE with mean, MHBFE with median and MHBFE with mode for (a) PSNR, (b) Entropy and (c) AMBE

Table 4: Goodness-of-fit measure

\begin{tabular}{|l|l|l|l|l|}
\hline Parameter & HBFE & $\begin{array}{l}\text { MHBFE } \\
\text { with mean }\end{array}$ & $\begin{array}{l}\text { MHBFE } \\
\text { with median }\end{array}$ & $\begin{array}{l}\text { MHBFE } \\
\text { with mode }\end{array}$ \\
\hline PSNR Goodness-of-fit & 0.980 & 0.957 & 0.910 & 0.892 \\
\hline Entropy Goodness-of-fit & 0.871 & 0.778 & 0.867 & 0.886 \\
\hline AMBE Goodness-of-fit & 0.577 & 0.378 & 0.481 & 0.507 \\
\hline
\end{tabular}

Another important parameter needed in the following statistical analysis is the population variance $\sigma^{2}$ which is a measure of how far each value in the data set is from the mean and it is measured as: 
Signal \& Image Processing : An International Journal (SIPIJ) Vol.5, No.1, February 2014

$$
\sigma^{2}=\sum_{i=1}^{N} p\left(x_{i}\right)\left(x_{i}-\mu\right)^{2}
$$

Where the distribution $P(x)$ with known population mean $\mu$.

The next step is the using of ANalysis Of VAriance (ANOVA). It will be used to test if the HBFE and MHBFE with its methods are similar, and it accurately find if one of them is different and show a significant difference between them. Its idea based on satisfying some assumptions:

1. Single quantitative response variable where we apply the test of each parameter for each method.

2. Independent groups, the data sample of CT images are not depend on each other.

3. SRS (Simple Random Sampling) is used to collect the data, this is satisfied by select images randomly without any condition.

4. Common variances for all groups. Calculation of the variance for all parameters of methods in Table 5, shows that they satisfy the condition [17]:

$$
\frac{\sigma_{\max }^{2}}{\sigma_{\min }^{2}} \leq 4
$$

5. Population Distribution of response variable is approximate normal for PSNR, entropy for all methods but the Population Distribution for AMBE make a little diverge as shown in figure 10 and table 4 but the usage of $100 \mathrm{CT}$ lung image as large number of images in the used sample overcome this point.

Table 5: variance measure

\begin{tabular}{|l|l|l|l|l|}
\hline Parameter & HBFE & $\begin{array}{l}\text { MHBFE } \\
\text { with mean }\end{array}$ & $\begin{array}{l}\text { MHBFE } \\
\text { with median }\end{array}$ & $\begin{array}{l}\text { MHBFE } \\
\text { with mode }\end{array}$ \\
\hline PSNR variance $\left(\sigma^{2}\right)$ & 58.12 & 63.87 & 59.51 & 63.3 \\
\hline Entropy variance $\left(\sigma^{2}\right)$ & 0.4667 & 0.6213 & 0.4771 & 0.4527 \\
\hline AMBE variance $\left(\sigma^{2}\right)$ & 61.55 & 127.9 & 54.28 & 47.34 \\
\hline
\end{tabular}

After satisfying these assumptions on the selected sample of data. We use t- test that is used to measure the confidence ratio which shows how the proposed modified algorithm achieves differences in the enhancement results. By applying this test we found the confidence ratios are different for each parameter and also for each modification method. These results are summarized in Table 6. 
Signal \& Image Processing : An International Journal (SIPIJ) Vol.5, No.1, February 2014

Table 6: The confidence ratio

\begin{tabular}{|l|l|l|l|}
\hline & Mean & Median & Mode \\
\hline PSNR & 0.897 & 0.998 & 0.999 \\
\hline entropy & 0.503 & 0.657 & 0.675 \\
\hline AMBE & 0.527 & 0.881 & 0.918 \\
\hline
\end{tabular}

As shown in Table 6, the best values of confidence ratio is PSNR of mode method, where its value is 0.999 .This means that if we have 1000 image we find only one image has the same value of PSNR using the mode modification like HBFE but the other 999 images have improvement in PSNR value [17]. If we make a simple comparison among confidence values, we find that there is high increase in PSNR, and this due to the high difference in the PSNR improvement that occurred using the three modification methods as illustrated before in section 4 , there is a moderate difference in the entropy values. The confidence levels for AMBE is also noticeable high for the mode method but is low for the mean method and this means there is a similarity between the AMBE of HBFE values and MFBHE using mean.

These statistical results are close in meaning to the previous experimental results, which it also shows that there is an improvement occurred in PSNR, entropy and AMBE. We can also conclude that the variance in results due to the characteristic of the image. By using the statistical analysis, We can exclude that there is variable enhancements depend on the image features, this make the proposed medication is more generalized for any image and not specialized for one type.

\section{CONCLUSION}

In this paper, we have presented simple three modifications of Histogram Based Fast Enhancement Algorithm. First, we have showed how it succeeded in removing water-washed effect. Then discuss the proposed modification which enhances the PSNR, AMBE and entropy parameters values to be more appropriate for a wide range of CT images. In addition to the enhancements occurred to the HBFE, There are some advantages of the algorithm compared to other algorithms. It still keeps the advantage of simplicity due to less complex calculations used in the algorithm. There is another advantage of this algorithm due to its idea of using global histogram and not based on local histogram. This decreases the used time for running. We also make statistical analysis for the modification to be generalized as enhancement technique.

\section{REFERENCES}

[1] L.Yang, Y.Liang \& H.Fan, (2010) " Study on the methods of image enhancement for liver CT images", International Journal for Light and Electron Optics, Volume 121, Issue 19, ISSN: 00304026, pp 1752-1755

[2] F. Taher, N.Werghi, H. Al-Ahmad \& R. Sammouda, (2012) "Lung Cancer Detection by Using Artificial Neural Network and Fuzzy Clustering Methods" American Journal of Biomedical Engineering, 2(3), ISSN: 2163-1050, pp136-142 
Signal \& Image Processing : An International Journal (SIPIJ) Vol.5, No.1, February 2014

[3] A.Chaudhary \& S.Singh, (2012) "LUNG CANCER DETECTION USING DIGITAL IMAGE PROCESSING", Volume 2, Issue 2, ISSN: 2249-3905, pp 1351-1359

[4] S. Smith, (2002) "The Scientist and Engineer's Guide to Digital Signal Processing", Chapter 25: Special Imaging Techniques, Computed Tomography, ISBN 0-7506-7444-X.

[5] D.Tsai, N.Takahashi \& Y. Lee, (2005)" An Adaptive Enhancement Algorithm for CT Brain Images", IEEE Engineering in Medicine and Biology 27th Annual Conference Shanghai, China

[6] Y. Kim, (1997) "Contrast enhancement using brightness preserving Bi-Histogram equalization", IEEE Trans. Consumer Electronics, vol. 43, no. 1, pp. 1-8.

[7] R. Krutsch \& D. Tenorio, (2011) "Histogram Equalization", Document Number: AN4318, Application Note Rev. 0.

[8] I. Jafar \& H. Ying, (2007) " Multilevel Component-Based Histogram Equalization for Enhancing the Quality of Grayscale Images", IEEE EIT, pp. 563-568.

[9] R. Jonesand \&T. Tjahjadi, (1993) "A study and Modification of the Local Histogram Equalization Algorithm", pattern recognition, vol2, no. 9, pp 1373-1381.

[10] P. Shanmugavadivu\& K. Balasubramania, (2010) "Image Inversion and Bi Level Histogram Equalization for Contrast Enhancement" International Journal of Computer Applications (0975 8887) Volume 1 - No. 15.

[11] A. Zadbuke, (2012)"Brightness Preserving Image Enhancement Using Modified Dualistic Sub Image Histogram Equalization", International Journal of Scientific \& Engineering Research, Volume 3, Issue 2, 1 ISSN 2229-5518.

[12] P. Jagatheeswari, S. Kumar\& M. Rajaram, (2009)" Contrast Enhancement for Medical Images Based on Histogram Equalization Followed by Median Filter", the International Conference on ManMachine Systems (ICoMMS), MALAYSIA.

[13] H. Cheng \& X. Shi, (2004)"A simple and effective histogram equalization approach to image enhancement", Digital Signal Processing, 14, ISSN: 1051-2004, 158-170

[14] J.Kim, L.Kim\& S.Hwang, (2001) "An Advanced Contrast Enhancement Using Partially Overlapped Sub-Block Histogram Equalization", IEEE TRANSACTIONS ON CIRCUITS AND SYSTEMS FOR VIDEO TECHNOLOGY, VOL. 11, NO. 4, pp 475-484

[15] J.Yin, X. Tian, Z. Tang \&Y. Sun, (2006) "A Histogram-Based Fast Enhancement Algorithm for CT Head Images", Intl. Conf. on Biomedical and Pharmaceutical Engineering (ICB PE).

[16] The crystallographic binary file, (2013)" Image entropy", http://www.esrf.eu/computing/forum/imgCIF/PAPER/entropy

[17] R. Lyman Ott, (2010) "An Introduction to Statistical Methods and Data Analysis", Sixth Edition, chapter 5: inferences about population central values, ISBN-10: 0-495-01758-2, pp 232-244

[18] Engineering Statistics, (2012) "Anderson-Darling and Shapiro-Wilk tests", http://www.itl.nist.gov/div898/handbook/prc/section2/prc213.htm 